\title{
Avaliação anatômica da valva tricúspide
}

Fábio B. JATENE*, Márcia Kiyomi KOIKE*, Rosângela MONTEIRO*, Siomara Christina VERONEZI*, Maria Helena G. MAGALHĀES*, Adib D. JATENE*

JATENE, F. B.; KOIKE, M. K.; MONTEIRO, R.; VERONEZI, S. C.; MAGALHÄES, M. H. G.; JATENE, A. D. - Avaliação anatômica da valva tricúspide. Rev. Bras. Cir. Cardiovasc., 7(1):22-27, 1992.

RESUMO: O objetivo deste estudo é avaliar dados anatômicos da valva tricuspide e região adjacente que possam auxiliar em procedimentos diagnósticos e cirúrgicos. Em 101 coraçöes humanos normais (84\% do sexo masculino, $61 \%$ do grupo étnico branco e com idades entre 9 e 86 anos $(30,0+15,5)$, foram analisados os seguintes aspectos: número de cúspides; perímetro do anel tricúspide; distância intercomissural interna e externa; porcentagem de ocupação das cúspides no anel; largura das cúspides e área do triângulo de Koch. Esses aspectos foram relacionados com idade, sexo e grupo étnico. Em nosso material, $73 \%$ das valvas apresentavam três cúspides, $26 \%$ duas e $1 \%$ quatro. O perímetro do anel tricúspide variou de $79 \mathrm{~mm}$ a 158 $\mathrm{mm}(109,6 \mathrm{~mm}+12,2 \mathrm{~mm})$, sendo $9 \%$ maior no sexo masculino. Com relaçäo à porcentagem de ocupação das cúspides no anel valvar, a cúspide anterior ocupou em média $48 \%$ do anel, a septal $36 \%$ e a posterior $16 \%$. A área média do triângulo de Koch foi $167,79 \mathrm{~mm}^{2}+71,88 \mathrm{~mm}^{2}$, sendo $10 \%$ maior no grupo étnico näo branco e em faixa etária superior a 30 anos. Em conclusão, valvas tricúspides normais apresentam variaçōes estruturais relacionadas com sexo, idade e grupo étnico.

DESCRITORES: valva tricuspide, anatomia.

\section{INTRODUÇÃO}

A literatura sobre anatomia e cirurgia da valva atrioventricular direita, também conhecida como valva tricúspide (VT), é pobre e apresenta pontos de vista muito divergentes ${ }^{8,12}$.

A descrição clássica da VT é que a mesma é constituída de três cúspides ou folhetos ${ }^{2,15}, 20,27$. Entretanto, os textos modernos de anatomia sugerem que o número de cúspides pode variar ${ }^{2,12}$, ${ }^{18,28}$ e que cúspides acessórias podem estar presentes entre as principais ${ }^{20}$.

A ausência de uma clara definição das comissuras - que normalmente não atingem o anel 6, 18 - e da extensão das cúspides dificulta a avaliação macroscópica e a padronização da nomenclatura dessas estruturas. Possivelmente, essa dificuldade tem persistido porque a comissurotomia da VT não é tão freqüentemente realizada $12,15,20$.

A cirurgia da VT é, dificilmente, um evento isolado; normalmente é feita em conjunto com a cirurgia da valva mitral e é, quase sempre, conseqüência do desenvolvimento da hipertensão pulmonar e do aumento do ventrículo direito resultando na dilatação do anel tricúspide 4, 9, 21.

Estudos anatômicos têm mostrado que a dilatação do anel não afeta as três cúspides no mesmo grau, sendo mais atingidas as cúspides anterior $e$ posterior ${ }^{1,6,7,9}$. A cúspide septal, que faz parte do esqueleto fibroso do coração, praticamente não sofre dilatação e é usada em plastias como limite para quantificar o diâmetro ótimo do anel valvar 6, 7, 11 .

O objetivo do presente estudo é a avaliação

Trabalho realizado no Museu Anatômico - Cirúrgico do Instituto do Coração do Hospital das Clínicas da Faculdade de Medicina da Universidade de São Paulo. São Paulo, SP, Brasil.

Recebido para publicação em 12 de fevereiro de 1992.

* Do Instituto do Coração do Hospital das Clínicas da Faculdade de Medicina da Universidade de São Paulo.

Endereço para separatas: Fábio Jatene. Av. Dr. Enéas de Carvalho Aguiar, 44. Divisão Cirúrgica. 05403 São Paulo, SP, Brasil. 
JATENE, F. B.; KOIKE, M. K.; MONTEIRO, R.; VERONEZI, S. C.; MAGALHÄES, M. H. G.; JATENE, A. D. - Avaliação anatômica da valva tricúspide. Rev. Bras. Cir. Cardiovasc., 7(1):22-27, 1992.

detalhada da anatomia da VT com ênfase em suas cúspides, visando fornecer dados que possam auxiliar em plastias e no implante de próteses de tamanho apropriado. Este trabalho busca, ainda, relação desses vários aspectos anatômicos com as variáveis grupo étnico, sexo e idade.

\section{MATERIAL E MÉTODOS}

Neste estudo foram utilizados 101 coraçōes humanos anatomicamente normais, previamente fixados, com massa entre 107 e 463 gramas $(259,5+$ $59,3) ; 84(84 \%)$ pertenciam ao sexo masculino. Quanto ao grupo étnico, 39 (39\%) eram de indivíduos não-brancos (pardos, negros e amarelos). A idade variou de 9 a 86 anos $(30,0+15,5)$.

Utilizando-se de um fio de algodão zero e de um paquímetro, foram realizadas as seguintes avaliaçōes na VT ao nível de inserção das cúspides e região adjacente:

a) número de cúspides;

b) distâncias intercomissurais externas, medidas pelas paredes da aorta, e distâncias intercomissurais internas, que representam retas ligando as comissuras na menor distância entre elas (Figura 1);

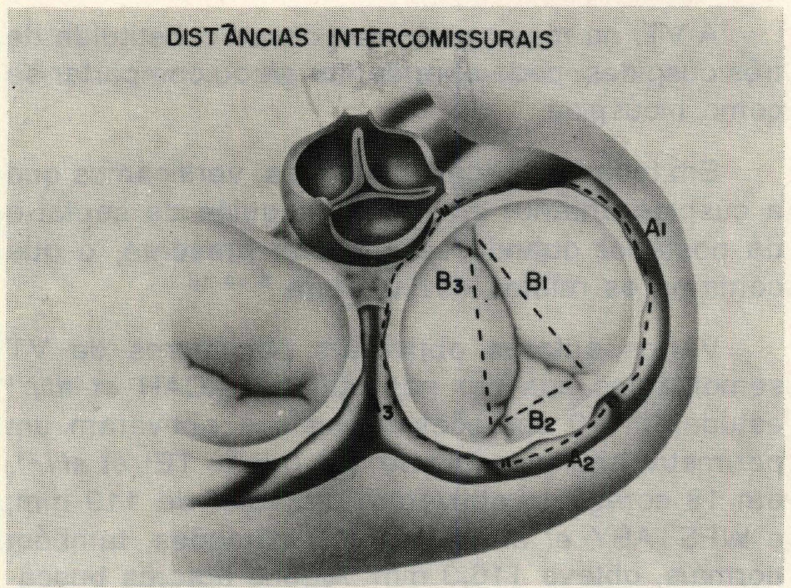

Fig. 1 -Esquema da valva tricúspide. $A=$ distâncias intercomissurais externas: $A 1$ = cúspide anterior; $A 2$ = cúspide posterior; $A 3$ = cúspide septal. $B=$ distâncias intercomissurais internas: $\mathrm{B} 1$ = cúspide anterior; $\mathrm{B} 2$ = cúspide posterior; B3 = cúspide septal.

c) perímetro do anel tricúspide, obtido pela soma das distâncias intercomissurais externas;

d) porcentagem de ocupação das cúspides no anel, calculada dividindo-se a distância intercomissural externa de cada cúspide pelo perímetro total do anel;

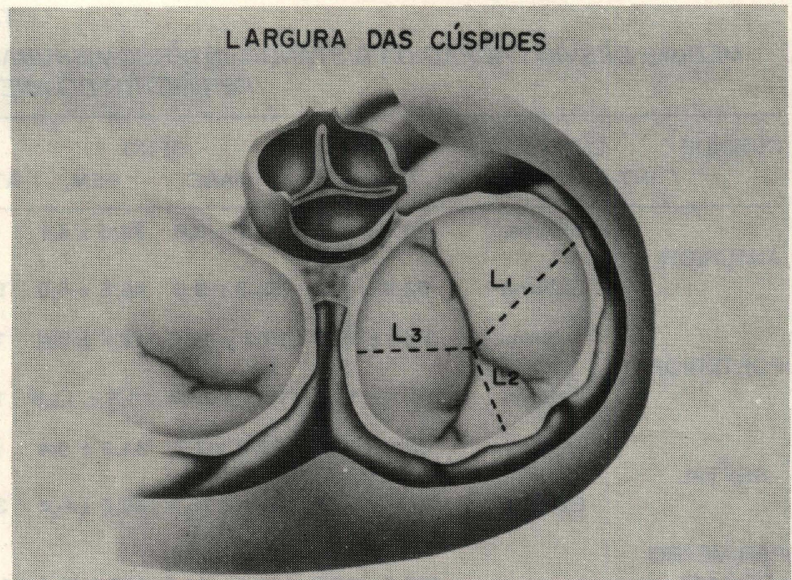

Fig. 2 - Esquema da valva tricúspide. $L$ = larguras das cúspides no ponto médio entre as comissuras: L1 = cúspide anterior; L2 - cúspide posterior; L3 = cúspide septal.

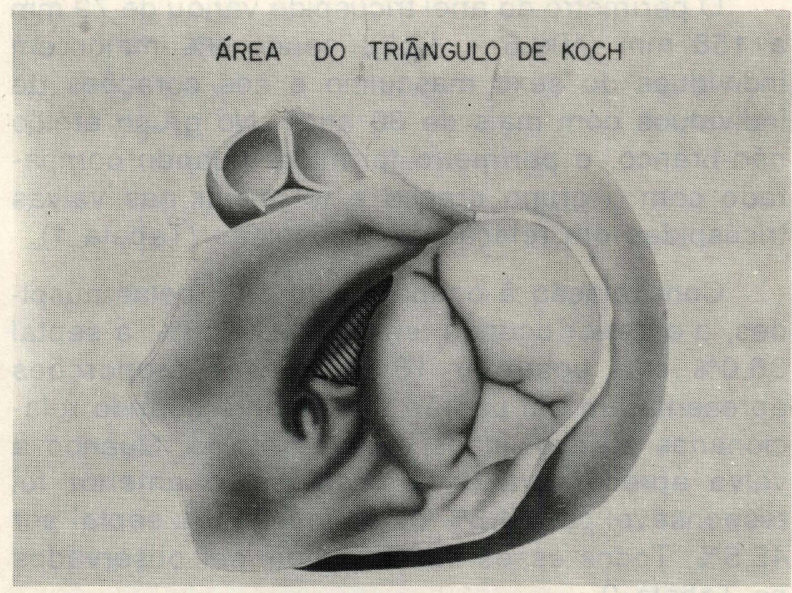

Fig. 3- Esquema da valva tricúspide e triângulo de Koch.

e) largura das cuspides no ponto médio entre as comissuras (Figura 2);

f) área do triângulo de Koch (Figura 3)

Todos os aspectos estudados foram relacionados com sexo, idade, grupo étnico e número de cúspides.

\section{RESULTADOS}

Dos corações estudados, $73 \%$ apresentavam valvas com três cúspides, $26 \%$ duas e $1 \%$ quatro, esta última com cúspide posterior dupla. Os dados referentes à valva quadricúspide foram excluídos para análise devido à sua baixa incidência.

Quanto às distâncias intercomissurais, interna e externa, a cúspide anterior apresentou as maiores distâncias, em todas as peças estudadas, seguida da cúspide septal e da posterior (Tabela 1). 
JATENE, F. B.; KOIKE, M. K.; MONTEIRO, R.; VERONEZI, S. C.; MAGALHÄES, M. H. G.; JATENE, A. D. - Avaliação anatômica da valva tricúspide. Rev. Bras. Cir. Cardiovasc., 7(1):22-27, 1992.

TABELA 1

MÉDIA E DESVIO PADRÃO DA DISTÂNCIA INTERCOMISSURAL INTERNA E EXTERNA DAS CÚSPIDES DA VALVA TRICÚSPIDE E DO PERIMETRO DO ANEL VALVAR TRICÚSPIDE (MM)

\begin{tabular}{|c|c|c|c|c|c|c|c|c|c|c|}
\hline \multirow{2}{*}{ CÚSPIDE } & \multirow{2}{*}{$\begin{array}{c}\text { DISTÂNCIA } \\
\text { INTERCOMISSURAI }\end{array}$} & \multirow{2}{*}{$\begin{array}{r}\text { MÉDIA } \\
\text { L GERAL }\end{array}$} & \multicolumn{2}{|c|}{ SEXO } & \multicolumn{2}{|c|}{ IDADE } & \multicolumn{2}{|c|}{ GRUPO ÉTNICO } & \multirow{2}{*}{\multicolumn{2}{|c|}{$\begin{array}{c}N^{2} D E \text { CÚSPIDES } \\
\text { BICÚSPIDE TRICÚSPIDE }\end{array}$}} \\
\hline & & & MASC. & FEM. & ATÉ 30 ANOS & $>30$ ANOS & BRANCO & NÄO-BRANCO & & \\
\hline \multirow{2}{*}{ ANTERIOR } & Interna & $38,0 \pm 6,5$ & $38,7 \pm 6,6$ & $34,5 \pm 4,7$ & $38,6 \pm 7,4$ & $37,1 \pm 4,6$ & $37,5 \pm 6,1$ & $38,8 \pm 7,1$ & $40,2 \pm 5,9$ & $36,8 \pm 5,8$ \\
\hline & Externa & $52,8 \pm 9,4$ & $53,3 \pm 9,6$ & $46,5 \pm 6,0$ & $53,4 \pm 10,2$ & $50,2 \pm 7,6$ & $51,0 \pm 10,0$ & $54,0 \pm 8,2$ & $57,6 \pm 7,4$ & $50,3 \pm 9,3$ \\
\hline \multirow{2}{*}{ POSTERIOR } & Interna & $14,8 \pm 10,7$ & $15,2 \pm 10,9$ & $12,7 \pm 9,8$ & $12,8 \pm 10,8$ & $17,9 \pm 10,0$ & $15,2 \pm 11,2$ & $13,9 \pm 9,8$ & - & $20,0 \pm 7,1$ \\
\hline & Externa & $18,1 \pm 13,0$ & $18,5 \pm 13,3$ & $15,6 \pm 11,5$ & $15,2 \pm 12,7$ & $22,4 \pm 12,3$ & $17,9 \pm 13,1$ & $18,2 \pm 12,2$ & - & $24,3 \pm 8,5$ \\
\hline \multirow{2}{*}{ SEPTAL } & Interna & $33,0 \pm 7,4$ & $33,2 \pm 7,7$ & $31,9 \pm 6,4$ & $33,6 \pm 8,2$ & $32,1 \pm 6,0$ & $32,9 \pm 7,7$ & $33,3 \pm 7,1$ & $39,4 \pm 5,2$ & $30,8 \pm 6,8$ \\
\hline & Externa & $39,5 \pm 10,6$ & $39,3 \pm 10,9$ & $40,0 \pm 9,4$ & $39,5 \pm 10,7$ & $39,4 \pm 10,6$ & $38,9 \pm 10,3$ & $40,3 \pm 11,1$ & $48,2 \pm 7,7$ & $36,4 \pm 9,7$ \\
\hline \multicolumn{11}{|l|}{ PERÍMETRO } \\
\hline $\begin{array}{l}\text { DO ANEL } \\
\text { TRICÚSPIDE }\end{array}$ & & $109,6 \pm 12,2$ & $111,1 \pm 11,8$ & $102,112,1$ & $108,1 \pm 12,9$ & $112,0 \pm 10,9$ & $107,9 \pm 11,8$ & $3112,41 \pm 12,5$ & $105,8 \pm 9,9$ & $111,0 \pm 12,7$ \\
\hline
\end{tabular}

O perímetro do anel tricúspide variou de $79 \mathrm{~mm}$ a $158 \mathrm{~mm}(109,6+12,2)$, sendo $9 \%$ maior em indivíduos do sexo masculino e nos corações de indivíduos com mais de 30 anos. No grupo étnico não-branco, o perímetro foi maior quando comparado com o grupo branco e foi maior nas valvas tricúspides em relação às bicúspides (Tabela 1).

Com relação à ocupação do anel pelas cúspides, a anterior ocupou, em média, $47,8 \%$, a septal $36,0 \%$ e a posterior $16,2 \%$. Essas proporções apresentaram um padrão semelhante quando relacionadas a sexo, idade e grupo étnico. Quando a valva apresentava duas cúspides, a anterior foi responsável por $54,5 \%$ de ocupação e a septal por $45,5 \%$. Todos esses dados podem ser observados na Tabela 2.

Com referência à largura das cúspides, na média, a anterior apresentou $20,2 \mathrm{~mm}+5,1 \mathrm{~mm}$, a septal $15,4 \mathrm{~mm}+2,7 \mathrm{~mm}$ e a posterior $10,5 \mathrm{~mm}$ $+7,7 \mathrm{~mm}$. Foi maior no sexo masculino, nos casos com idade acima de 30 anos e no grupo étnico não-branco. Quando a valva foi bicúspide, a largura da cúspide anterior foi $18,5 \mathrm{~mm}+3,9 \mathrm{~mm}$ e a septal $15,3 \mathrm{~mm}+3,1 \mathrm{~mm}$. Todos os valores podem ser observados na Tabela 3.

Com relação à área do triângulo de Koch, ela foi em média de $167,79 \mathrm{~mm}^{2}+71,88 \mathrm{~mm}^{2}$, sendo maior no sexo feminino, nos coraçōes de indivíduos com mais de 30 anos, no grupo étnico não-branco e nos corações com valvas tricúspides. O detaIhamento desses valores, bem como dos valores dos lados do triângulo de Koch para cada uma das variáveis analisadas está representado na Tabela 4.

\section{COMENTÁRIOS}

A VT, na maior parte dos. casos constituída de três cúspides, pode aprersentar-se ou comportar-se como bicúspide.

Em todas as peças estudadas, verificamos que a cúspide anterior era maior, seguida da septal e da posterior quando esta estava presente, o que confirma os relatos da literatura $5,19,15$.

Vários autores obtiveram perímetros da VT semelhantes aos por nós obtidos. ACAR et alii ${ }^{1}$ estudaram 10 corações normais e obtiveram um perímetro anular médio de $114,0 \mathrm{~mm}$; TEl et alii ${ }^{24}$, em 18 corações, obtiveram um valor de $119 \mathrm{~mm}$; e WESTABY et alii ${ }^{26}$, em 160 corações, também normais, obteve $116,3 \mathrm{~mm}$. Alguns autores buscaram, ainda, relação entre perímetro anular e sexo.

TABELA 2

PORCENTAGEM DE OCUPAÇÃO DAS CÚSPIDES NO ANEL VALVAR TRICÚSPIDE

\begin{tabular}{|c|c|c|c|c|c|c|c|c|c|}
\hline \multirow[t]{2}{*}{ CÚSPIDE } & \multirow{2}{*}{$\begin{array}{l}\text { MÉDIA } \\
\text { GERAL }\end{array}$} & \multicolumn{2}{|c|}{ SEXO } & \multicolumn{2}{|c|}{ IDADE } & \multicolumn{2}{|c|}{ GRUPO ÉTNICO } & \multirow{2}{*}{\multicolumn{2}{|c|}{$\begin{array}{c}\text { Ne DE CÚSPIDES } \\
\text { BICÚSPIDE TRICÚSPIDE }\end{array}$}} \\
\hline & & MASCULINO & FEMININO & ATÉ 30 ANOS & $>30$ ANOS & BRANCO & NÃO-BRANCO & & \\
\hline ANTERIOR & $47,8 \pm 8,1$ & $48,2 \pm 8,2$ & $46,0 \pm 7,0$ & $49,5 \pm 8,2$ & $45,1 \pm 7,1$ & $47,5 \pm 8,4$ & $48,3 \pm 7,5$ & $54,5 \pm 5,4$ & $45,5 \pm 7,5$ \\
\hline POSTERIOR & $16,2 \pm 11,3$ & $16,4 \pm 11,4$ & $14,8 \pm 10,7$ & $14,0 \pm 11,4$ & $19,6 \pm 10,4$ & $16,3 \pm 11,7$ & $15,9 \pm 10,8$ & - & $21,9 \pm 6,8$ \\
\hline SEPTAL & $36,0 \pm 9,0$ & $35,4 \pm 9,0$ & $39,3 \pm 8,4$ & $39,5 \pm 9,0$ & $35,3 \pm 9,1$ & $36,2 \pm 9,3$ & $35,8 \pm 8,7$ & $45,5 \pm 5,3$ & $32,7 \pm 7,7$ \\
\hline
\end{tabular}


JATENE, F. B.; KOIKE, M. K.; MONTEIRO, R.; VERONEZI, S. C.; MAGALHÄES, M. H. G.; JATENE, A. D. - Avaliação anatômica da valva tricúspide. Rev. Bras. Cir. Cardiovasc., 7(1):22-27, 1992.

TABELA 3

MÉDIA E DESVIO PADRÃO DA LARGURA DAS CÚSPIDES DA VALVA TRICÚSPIDE (MM).

\begin{tabular}{|c|c|c|c|c|c|c|c|c|c|}
\hline \multirow[t]{2}{*}{ CÚSPIDE } & \multirow{2}{*}{$\begin{array}{l}\text { MÉDIA } \\
\text { GERAL }\end{array}$} & \multicolumn{2}{|c|}{ SEXO } & \multicolumn{2}{|c|}{ IDADE } & \multicolumn{2}{|c|}{ GRUPO ÉTNICO } & \multirow{2}{*}{\multicolumn{2}{|c|}{$\begin{array}{l}N^{\circ} \text { DE CÚSPIDES } \\
\text { BICÚSPIDE TRICÚSPIDE }\end{array}$}} \\
\hline & & MASCULINO & FEMININO & ATÉ 30 ANOS & $>30$ ANOS & BRANCO & NÃO-BRANCO & & \\
\hline ANTERIOR & $20,2 \pm 5,1$ & $20,3 \pm 4,3$ & $18,1 \pm 4,8$ & $19,2 \pm 4,6$ & $21,1 \pm 4,0$ & $19,5 \pm 4,9$ & $20,7 \pm 3,7$ & $18,5 \pm 3,9$ & $20,5 \pm 4,5$ \\
\hline POSTERIOR & $10,5 \pm 7,7$ & $10,3 \pm 7,6$ & $11,1 \pm 7,9$ & $9,3 \pm 7,7$ & $12,5 \pm 7,3$ & $10,0 \pm 7,6$ & $11,2 \pm 7,7$ & - & $15,4 \pm 11,7$ \\
\hline SEPTAL & $15,4 \pm 2,7$ & $15,5 \pm 2,5$ & $14,6 \pm 3,7$ & $15,1 \pm 2,8$ & $15,8 \pm 2,6$ & $14,9 \pm 2,7$ & $16,08 \pm 2,8$ & $15,3 \pm 3,1$ & $15,4 \pm 2,7$ \\
\hline
\end{tabular}

TABELA 4

MÉDIA E DESVIO PADRÃO DA ÁREA DO TRIÂNGULO DE KOCH (MM²) E SEUS LADOS (MM)

\begin{tabular}{|c|c|c|c|c|c|c|c|c|c|}
\hline & \multirow{2}{*}{$\begin{array}{l}\text { MÉDIA } \\
\text { GERAL }\end{array}$} & \multicolumn{2}{|c|}{ SEXO } & \multicolumn{2}{|c|}{ IDADE } & \multicolumn{2}{|c|}{ GRUPO ÉTNICO } & \multicolumn{2}{|c|}{ N DE CÚSPIDES } \\
\hline & & MASCULINO & FEMININO & ATÉ 30 ANOS & $>30$ ANOS & BRANCO & NÃO-BRANCO & BICÚSPIDE & TRICÚSPIDE \\
\hline ÁREA & $167,8 \pm 71,9$ & $167,5 \pm 68,4$ & $169,4 \pm 90,4$ & $161,2 \pm 59,6$ & $178,0 \pm 87,7$ & $161,5 \pm 59,8$ & $177,7 \pm 87,5$ & $159,6 \pm 56,9$ & $169,5 \pm 78,4$ \\
\hline & $13,6 \pm 4,2$ & $13,6 \pm 3,9$ & $14,0 \pm-5,6$ & $13,3 \pm 3,9$ & $14,1 \pm 4,7$ & $13,3 \pm 4,0$ & $14,2 \pm 4,5$ & $12,7 \pm 4,1$ & $14,1 \pm 4,2$ \\
\hline sL & $30,1 \pm 5,2$ & $30,2 \pm 5,0$ & $30,0 \pm 6,2$ & $29,4 \pm 5,2$ & $31,2 \pm 4,9$ & $30,5 \pm 6,2$ & $29,9 \pm 4,4$ & $29,7 \pm 4,5$ & $30,3 \pm 5,4$ \\
\hline
\end{tabular}

SILVER et alii ${ }^{20}$, em 50 corações normais, obtiveram uma média de $114 \mathrm{~mm}$ no sexo masculino e $108 \mathrm{~mm}$ no sexo feminino; WESTABY et alii ${ }^{26}$, em 160 coraçōes, obtiveram média de $119,5 \mathrm{~mm}$ no sexo masculino e $104,0 \mathrm{~mm}$ no feminino. Em nossos resultados, o perímetro anular médio foi 109,6 $\mathrm{mm}$, a média do sexo masculino foi $111,1 \mathrm{~mm}$ e $102,1 \mathrm{~mm}$ no sexo feminino.

A relação entre perímetro anular e sexo é consistente. Como esperado, em nosso estudo o perímetro da VT no sexo masculino foi cerca de $9 \%$ maior que no sexo feminino, o que converge com os trabalhos de WESTABY et alii ${ }^{26}$, que relatam uma diferença de $10 \%$ no tamanho das valvas entre os sexos, com predominância do sexo masculino.

Com relação à largura das cúspides, os resultados de SILVER et alii ${ }^{20}$ assemelharam-se aos nossos. Em seu trabalho, a cúspide anterior apresentou largura de $24 \mathrm{~mm}$ no sexo masculino e 21 $\mathrm{mm}$ no feminino; a cúspide septal apresentou largura de $17 \mathrm{~mm}$ no sexo masculino e $15 \mathrm{~mm}$ no feminino. Em nosso estudo, a cúspide anterior apresentou largura de $20,3 \mathrm{~mm}$ no sexo masculino e $18,1 \mathrm{~mm}$ no sexo feminino e a cúspide septal apresentou $15,5 \mathrm{~mm}$ no sexo masculino e $14,6 \mathrm{~mm}$ no feminino. Uma comparação relativa à cúspide posterior não foi possível, pois adotamos diferentes critérios na avaliação do mesmo.

Existe muita divergência na literatura em relação à técnica a ser empregada para correção da insuficiência tricúspide $3,5,7,9,12,16,17,22$.
As complicaçōes que advêm da colocação de próteses como tromboembolismo, endocardite, calcificação, roturas e complicaçōes mecânicas têm limitado sua aplicabilidade $7,19,21,23,25$. Alguns autores acreditam que as características hemodinâmicas das próteses em posição tricúspide contribuem para a alta incidência de baixo fluxo e conseqüente alta mortalidade hospitalar ${ }^{3}$. Em função disto, as plastias representam uma alternativa atraente, sendo utilizadas com grande freqüência nos vários centros de cirurgia cardíaca 3, 17, 22

Uma das primeiras técnicas de anuloplastia para correção da insuficiência tricúspide foi proposta por KAY et alii ${ }^{16}$, em 1965, onde são passadas duas suturas da comissura ântero-posterior à comissura póstero-septal, transformando a valva tricúspide em bicúspide. Essa técnica encontra respaldo anatômico, uma vez que resseca a cúspide posterior que, além de menor, normalmente é a que apresenta maior grau de distensão anular ${ }^{1}$. Além disso, com certa freqüência, a VT apresenta duas cúspides; em nosso material, verificamos que $26 \%$ das peças eram bicúspides. Entratanto, a técnica proposta por KAY et alii ${ }^{16}$ sofre algumas críticas em relação aos resultados tardios, uma vez que estudos anatomopatológicos mostram que neste tipo de anuloplastia o processo de dilatação anular continua, particularmente ao nível da cúspide anterior 9,22

Outras técnicas de plastia de VT foram propostas, sendo as mais utilizadas o uso do anel de Carpentier (CARPENTIER et alii ${ }^{7}$ ) e a anuloplastia proposta por De Vega (GRONDIN et alii ${ }^{{ }^{33}}$ ). 
JATENE, F. B.; KOIKE, M. K.; MONTEIRO, R.; VERONEZI, S. C.; MAGALHÄES, M. H. G.; JATENE, A. D. - Avaliaçäo anatômica da valva tricúspide. Rev. Bras. Cir. Cardiovasc., 7(1):22-27, 1992.

A plástica da VT pela técnica de De Vega apresenta várias vantagens, pois visa corrigir a insuficiência tricúspide atuando somente na alteração morfológica, constringindo apenas a região mais comprometida pelo processo de dilatação do anel, preservando a região correspondente à cúspide septal. A preservação da região septal é muito importante ${ }^{3}$ devido à proximidade com o triângulo de Koch, já que o feixe de His apresenta uma estreita relação com a região de fixação atrial da cúspide septal ${ }^{7,12}$; dessa forma esta técnica evita possíveis lesōes no sistema de condução ${ }^{9}, 13$.

Vários autores têm apresentado resultados favoráveis com a utilização do anel de Carpentier ${ }^{1,3,7 .}$ 13. O anel de Carpentier é adaptado à configuração anatômica do anel tricúspide; esta técnica permite restituição da circunferência normal por uma diminuição seletiva de segmentos do anel preferen- cialmente dilatado pelo processo patológico ${ }^{1}$. Além disso, ela também restaura a forma do orifício valvar 6,7 . Apesar dos resultados favoráveis, há desvantagens inerentes ao seu emprego, como a introdução de material sintético rígido e distúrbios de condução atrioventricular. Esse distúrbio ocorre porque o anel metálico pode funcionar como gerador de um campo eletromagnético provocando interferências eletromagnéticas no sistema de condução localizado nas proximidades do triângulo de Koch ${ }^{13,14}$.

A plastia de De Vega não apresenta esse problema, pois elimina a inserção de material estranho e é livre do risco de produzir um bloqueio atrioventricular por não requerer suturas na porção septal da valva ${ }^{7,14}$.

Em conclusão, valvas tricúspides normais apresentam variaçōes estruturais relacionadas com sexo, idade e grupo étnico.

\section{RBCCV 44205-158}

JATENE, F. B.; KOIKE, M. K.; MONTEIRO, R.; VERONESI, S. C.; MAGALHĀES, M. H. G.; JATENE, A. D. - Anatomic evaluation of the tricuspid valve. Rev. Bras. Cir. Cardiovasc., 7(1):22-27, 1992.

ABSTRACT: The purpose of this study is to evaluate anatomic data of the tricuspid valve and adjacent area to help in diagnostic and surgical procedures. In 101 normal human fixed hearts $(84 \%$ male; $61 \%$ white ethnic group and mean age of $30.0+15.5$ years old), we analysed: number of leaflets; tricuspid ring perimeter; size of leaflets and its occupation in ring perimeter; Koch's triangle area. These aspects were related with age, sex and ethnic group. There were $73 \%$ with three, in $26 \%$ with two and in $1 \%$ with four leaflets. The ring perimeter changed 79 to $158 \mathrm{~mm}(109.6+12.2)$ and was larger $(9 \%)$ in man. Regarding the relative occupation on ring the anterior represented $48 \%$, the septal $36 \%$ and the inferior $16 \%$ of occupation area in tricuspid ring. The mean area of Koch's triangle was $167.79 \mathrm{~mm}^{2}+71.88 \mathrm{~mm}^{2}, 10 \%$ larger in non white ethnic group and in elderly patients. In conclusion, normal tricuspid valves presented variations related to sex, age and ethnic group.

DESCRIPTORS: tricuspid valve, anatomy.

\section{REFERÊNCIAS BIBLIOGRÁFICAS}

1 ACAR, C.; PÉRIER, P.; FONTALIRAN, F.; DELOCHE, A.; CARPENTIER, A. - Anatomical study of the tricuspid valve and its variations. Surg. Radiol. Anat., 12: 229-233, 1990.

2 ANDERSON, R. H. \& BECKER, A. E. - Cardiac anatomy: an integrated text and colour atlas. London, Gower Medical Publishing, 1980. Cap.3, p. 3.2-3.3.

3 BOYD, A. D.; ENGELMAN, R. M.; ISOM, O. W.; REED, G. E.; SPENCER, F. C. - Tricuspid annuloplasty. J. Thorac. Cardiovasc. Surg., 68: 344-351, 1974.

BRAUNWALD, N. S.; ROSS Jr. J.; MORROW, A. G. Conservative management of tricuspid regurgitation in patients undergoing mitral valve replacement. Circulation, 35 (Supl. 1): 63-69, 1967.

5 BREYER, R. H.; McCLENATHAN, J. H.; MICHAELIS, L. L.; McINTOSH, C. L.; MORROW, A. G. - Tricuspid regurgitation. J. Thorac. Cardiovasc. Surg., 72: 867-874, 1976.

6 CARPENTIER, A. - Cardiac valve surgery: the "french correction". J. Thorac. Cardiovasc. Surg., 86: 323337, 1983.

7 CARPENTIER, A.; DELOCHE, A.; HANANIA, G.; FORMAN, J.; SELLIER, P.; PIWNICA, A.; DUBOST, C. - Surgical management of acquired tricuspid valve disease. J. Thorac. Cardiovasc. Surg., 67 (Supl.1): 53-65, 1974. 
JATENE, F. B.; KOIKE, M. K.; MONTEIRO, R.; VERONEZI, S. C.; MAGALHĀES, M. H. G.; JATENE, A. D. - Avaliação anatômica da valva tricúspide. Rev. Bras. Cir. Cardiovasc., 7(1):22-27, 1992.

8 CARVALHO, J. L. - Resultados tardios na substituição da valva tricúspide por valva de dura-máter homóloga. Salvador, 1975. [Tese. LivreDocência. Faculdade de Medicina da Universidade da Bahia].

9 DELOCHE, A.; GUÉRINON, J.; FABIANI, J. N. MORILLO, F.; CARAMANIAN, M.; CARPENTIER A.; MAURICE, P.; DUBOST, C. - Étude anatomique des valvopathies rhumatismales tricuspidiennes: application à l'étude critique des différentes méthodes d'annuloplastie. Arch. Mal. Coeur., 67: 497-506, 1973.

FRANKL, W. S. \& BREST, A. N. - Valvular heart disease: comprehensive evaluation and management. Philadelphia, Davis Co., 1986. p. 443-444.

GREENBERG, B. H. \& MURPHY, E. - Valvular heart disease. Massachussetts, PSG Publishing Co. 1987. p.56.

GRONDIN, P.; LEPAGE, G.; CASTONGUAY, Y.; MEERE, C.; - The tricuspid valve: a surgical challenge. J. Thorac. Cardiovasc. Surg., 53 (Supl. 1): 7-17, 1967.

GRONDIN, P.; MEERE, C.; LIMET, R.; LOPEZBESCOS, L.; DELCAN, J. L.; RIVERA, R. - Carpentier's annulus and De Vega's annuloplasty. $J$. Thorac. Cardiovasc. Surg., 70: 852-861, 1975.

HIGASHIDATE, M.; TAMIYA, K.; KUROSAWA, $\mathrm{H}$.; TAKANASHI, Y.; IMAI, Y. Real-time measurement of tricuspid valve annular area for annuloplasty: experimental study. J. Thorac. Cardiovasc. Surg., 96: 88-91, 1988.

HURST, J. W.; LOGUE, R. B.; RACKLEY, C. E.; SCHLANT, R. C.; SONNENBLICK, E. H.; WALLACE, A. G.; WENGER, N. K. - The heart: arteries and veins. New York, McGraw-Hill Book Co., 1985. p. 792-800.

16 KAY, J. H.; MASELLI-CAMPAGNA, G.; TOUGJ, H. K. - Surgical treatment of tricuspid insufficiency. Ann. Surg., 162: 53-58, 1965.

17 MINALE, C.; LAMBERTZ, H.; WIKOL, S.; GERICH, N.; MESSMER, B. J. - Selective annuloplasty of the

tricuspid valve. J. Thorac. Cardiovasc. Surg., 99: $846-851,1990$

18

NETTER, F. H. - Coração. Rio de Janeiro, Guanabara Koogan, 1976. p.6-14. (The Ciba Collection of Surgical Illustration, v.5).

STOLF, N.; MOREIRA, L. F. P.; COSTA, R.; BITTENCOURT, D.; VERGINELLI, G.; MACRUZ, R.; PILEGGI, F.; PUIG, L. B.; ZERBINI, E. J. - Tratamento cirúrgico conservador na insuficiência tricúspide funcional. Rev. Hosp. Clin. Fac. Med. S. Paulo, 36 (Supl.4): 146-151, 1981.

SYRACUSE, D. C.; BOWMAN Jr., F. O.; MALM, J. R. - Prosthetic valve reoperations. J. Thoracic. Cardiovasc. Surg., 77: 346-354, 1979.

24 TEI, C.; PILGRIM, J. P.; SHAH, P. M.; ORMISTON, J. A.; WONG, M. - The tricuspid valve annulus: study of size and motion in mormal subjects and in patients with tricuspid regurgitation. Circulation, 66: 665$671,1982$.

25 VAN DER VEER, J.; RHYNEER, G.; HODAM, R.; KLOSTER, F. E. - Obstruction of tricuspid ball-valve prostheses. Circulation, 43 (Supl. 1): 62-67, 1971.

26 WESTABY, S.; KARP, R. B.; BLACKSTONE, E. H.; BISHOP, S. P. - Adult human valve dimensions and their surgical significance. Am. J. Cardiol., 53: 552$556,1984$.

27 WILCOX, B. R. \& ANDERSON, R. H. - Surgical anatomy of the heart. London, Gower Medical Publishing Co., 1985. Cap. 2. p. 2.8-2.10.

28 ZEREN, Z. - Un cas de valve quinticuspide. Acta Anat., 13: 384-397, 1951. 